\title{
Balamuthia mandrillaris interactions with human brain microvascular endothelial cells in vitro
}

Correspondence

Naveed Ahmed Khan

n.khan@sbc.bbk.ac.uk

Received 21 December 2006

Accepted 23 April 2007

\author{
Abdul Matin, ${ }^{1}$ Ruqaiyyah Siddiqui, ${ }^{1}$ Suk-Yul Jung, ${ }^{1}$ Kwang Sik Kim, ${ }^{2}$ \\ Monique Stins ${ }^{2}$ and Naveed Ahmed Khan ${ }^{1}$
${ }^{1}$ School of Biological and Chemical Sciences, Birkbeck, University of London, London WC1E 7HX, UK MD, USA \\ ${ }^{2}$ Division of Pediatric Infectious Diseases, Johns Hopkins University School of Medicine, Baltimore,
}

\begin{abstract}
Balamuthia amoebic encephalitis (BAE) is a serious human disease almost always leading to death. An important step in BAE is amoebae invasion of the bloodstream, followed by their haematogenous spread. Balamuthia mandrillaris entry into the central nervous system most likely occurs at the blood-brain barrier sites. Using human brain microvascular endothelial cells (HBMECs), which constitute the blood-brain barrier, this study determined (i) the ability of $B$. mandrillaris to bind to HBMECs and (ii) the associated molecular mechanisms. Adhesion assays revealed that $B$. mandrillaris exhibited greater than $90 \%$ binding to HBMECs in vitro. To determine whether recognition of carbohydrate moieties on the surface of the HBMECs plays a role in $B$. mandrillaris adherence to the target cells, adhesion assays were performed in the presence of the saccharides mannose, galactose, xylose, glucose and fucose. It was observed that adherence of $B$. mandrillaris was significantly reduced by galactose, whilst the other saccharides had no effect. Acetone fixation of amoebae, but not of HBMECs, abolished adhesion, suggesting that $B$. mandrillaris adhesin(s) bind to galactose-containing glycoproteins of HBMECs. B. mandrillaris also bound to microtitre wells coated with galactose-BSA. By affinity chromatography using a galactose-Sepharose column, a galactose-binding protein (GBP) was isolated from detergent extracts of unlabelled amoebae. The isolation of a GBP from cell-surface-biotin-labelled amoebae suggested its membrane association. One-dimensional SDS-PAGE confirmed the proteinaceous nature of the GBP and determined its molecular mass as approximately $100 \mathrm{kDa}$. This is the first report suggesting the role of a GBP in $B$. mandrillaris interactions with HBMECs.
\end{abstract}

\section{INTRODUCTION}

Balamuthia mandrillaris is an emerging protozoan pathogen that can cause life-threatening infections involving the central nervous system (Schuster \& Visvesvara, 2004; Visvesvara et al., 1990, 1993). Balamuthia amoebic encephalitis (BAE) is characterized by headache, fever, characteristic skin lesions, stiff neck, nausea, vomiting, an acute confused state, cranial nerve palsies, seizures and finally death (Jayasekera et al., 2004; Schuster \& Visvesvara, 2004). Although there have been advances in the diagnosis of BAE (Booton et al., 2003a, b; Huang et al., 1999; Qvarnstrom et al., 2006; Tavares et al., 2006; Yagi et al., 2005), the pathogenesis and pathophysiology of this disease remain incompletely understood. Perhaps the most distressing aspect is the limited availability of effective

Abbreviations: BAE, Balamuthia amoebic encephalitis; GBP, galactosebinding protein; HBMEC, human brain microvascular endothelial cell; $\mathrm{LDH}$, lactate dehydrogenase. and/or recommended treatments against BAE. The infectious process involves amoebae invasion of the intravascular space through either the respiratory tract or skin lesions, followed by haematogenous spread. B. mandrillaris entry into the central nervous system most likely occurs at sites of the blood-brain barrier (Martinez et al., 2001; Schuster \& Visvesvara, 2004). However, the precise mechanisms by which $B$. mandrillaris transmigrates the blood-brain barrier are unclear. Using human brain microvascular endothelial cells (HBMECs), which constitute the blood-brain barrier, we have recently shown that B. mandrillaris produces severe host-cell cytotoxicity (Jayasekera et al., 2004; Matin et al., 2006, 2007). However, B. mandrillaris-mediated HBMEC cytotoxicity is a delayed event and requires parasite incubation with the host cells for over $24 \mathrm{~h}$. Here, we studied B. mandrillaris primary attachment to HBMECs and attempted to identify the associated molecular mechanisms. The carbohydrate moieties of the host-cell plasma membrane are major 
adhesion determinants for a number of pathogens including bacterial, fungal and protozoan pathogens (Alsam et al., 2003; Garate et al., 2006; Hostetter, 1994; Karlsson, 1989). Of interest, recent studies have shown that adhesion of $B$. mandrillaris to the extracellular matrix protein laminin can be blocked using exogenous galactose (Rocha-Azevedo et al., 2007). In the present study, we revealed that $B$. mandrillaris binds to HBMECs and demonstrated the presence of a galactose-binding protein (GBP) on the surface membrane of $B$. mandrillaris.

\section{METHODS}

Culture of B. mandrillaris. B. mandrillaris isolated from the brain of a mandrill baboon (ATCC 50209) was routinely cultured as described previously (Jayasekera et al., 2004; Matin et al., 2006). Briefly, B. mandrillaris was inoculated $\left(10^{6}\right.$ parasites) on confluent host-cell monolayers grown in T-75 tissue culture flasks. The amoebae consumed HBMECs within $48 \mathrm{~h}$ and produced approximately $5 \times 10^{6}-8 \times 10^{6}$ parasites ( $>99 \%$ in trophozoite form), which were used for subsequent experiments.

Cultures of HBMECs. Primary HBMECs were grown in RPMI 1640 with $20 \%$ heat-inactivated fetal bovine serum, $2 \mathrm{mM}$ glutamine, $1 \mathrm{mM}$ pyruvate, penicillin $\left(100 \mathrm{U} \mathrm{ml}^{-1}\right)$, streptomycin $\left(100 \mu \mathrm{g} \mathrm{ml}^{-1}\right)$, non-essential amino acids and vitamins as described previously (Alsam et al., 2003; Stins et al., 1997).

Adhesion assays. To determine the effects of saccharides on $B$. mandrillaris binding to HBMECs, adhesion assays were performed in the presence of various saccharides as described previously (Sissons et al., 2006). Briefly, HBMECs were grown in 24 -well plates by inoculating $5 \times 10^{5}$ cells per well and the plates were incubated at $37{ }^{\circ} \mathrm{C}$ in a $5 \% \mathrm{CO}_{2}$ incubator. Once confluent, HBMEC monolayers were incubated with $B$. mandrillaris $\left(5 \times 10^{5}\right.$ amoebae per well) in serum-free medium (RPMI 1640 containing $2 \mathrm{mM}$ glutamine, $1 \mathrm{mM}$ pyruvate and non-essential amino acids) and the plates were incubated at $37{ }^{\circ} \mathrm{C}$ in a $5 \% \mathrm{CO}_{2}$ incubator for $1 \mathrm{~h}$. After incubation, unbound amoebae were counted using a haemocytometer and the number of bound amoebae was calculated as follows: \% unbound amoebae $=$ (no. of unbound amoebae/total number of amoebae $) \times 100$. The number of bound amoebae was deduced as follows: $\%$ bound amoebae $=100-\%$ unbound amoebae. To test the effect of saccharides, $2 \times 10^{5}$ amoebae were incubated with various saccharides $[\alpha$-D-mannopyranoside ( $\alpha$-mannose), xylose, glucose, fucose and $\beta$ galactose at final concentrations of 10,50 and $100 \mathrm{mM}$ ] in $100 \mu \mathrm{l}$ RPMI 1640 for 30 min prior to the adhesion assay. In some adhesion assays, B. mandrillaris or HBMECs were fixed with $100 \%$ ice-cold acetone prior to adhesion assays (Jayasekera et al., 2004). In addition, 24-well plates were coated with galactose-BSA and mannose-BSA $\left(1 \mu \mathrm{g} \mathrm{ml}^{-1}\right.$ final concn) in coating buffer $(35 \mathrm{mM} \mathrm{NaHCO}, 15 \mathrm{mM}$ $\mathrm{Na}_{2} \mathrm{CO}_{3}$ ) and incubated for $24 \mathrm{~h}$ at $4{ }^{\circ} \mathrm{C}$. After incubation, wells were washed with $\mathrm{PBS}$ and inoculated with $B$. mandrillaris $\left(5 \times 10^{5}\right.$ amoebae per well). Plates were incubated at $37^{\circ} \mathrm{C}$ for $1 \mathrm{~h}$ to allow parasite binding. Finally, wells were washed with PBS and observed under a microscope.

Cytotoxicity assays. To determine the effects of saccharides on $B$. mandrillaris-mediated HBMEC death, cytotoxicity assays were performed as described previously (Kiderlen et al., 2006; Sissons et al., 2005). Briefly, B. mandrillaris was incubated with HBMECs as described for the adhesion assays and the plates were incubated for up to $24 \mathrm{~h}$. At the end of this incubation period, supernatants were collected and the cytotoxicity was determined by measuring lactate dehydrogenase (LDH) release using a cytotoxicity detection kit (Roche Applied Science). Briefly, conditioned medium of co-cultures of B. mandrillaris and HBMECs was collected and the percentage of LDH was detected as follows: $\%$ cytotoxicity $=$ (test value - control value $) /($ total $\mathrm{LDH}$ release - control value $) \times 100$. Control values were obtained from HBMECs incubated alone and total LDH release was measured from HBMECs treated with $5 \%$ Triton X-100 for $1 \mathrm{~h}$ at $37^{\circ} \mathrm{C}$.

Isolation of the GBP from B. mandrillaris trophozoites. To isolate the GBP of $B$. mandrillaris, cell pellets ( $10^{8}$ trophozoites) were washed three times in $20 \mathrm{ml}$ wash buffer $[50 \mathrm{mM}$ Tris/ $\mathrm{HCl}(\mathrm{pH} \mathrm{7.2)}$, $150 \mathrm{mM} \mathrm{NaCl}, 50 \mathrm{mM} \mathrm{CaCl}_{2}, 1 \mathrm{mM}$ PMSF] as described previously (Yang et al., 1997). The cell pellets were then resuspended in lysis buffer (wash buffer plus $0.5 \%$ CHAPS and $2 \mathrm{mM} \beta$-mercaptoethanol) and disrupted with a sonifier (model 250; Branson Ultrasonics) using a tapered microtip at an output setting of $20 \mathrm{~W}$. The amoebae extracts were clarified by centrifugation at $50000 \mathrm{~g}$ for $1 \mathrm{~h}$ at $4{ }^{\circ} \mathrm{C}$, followed by chromatography on a galactose-Sepharose column. Unbound proteins were removed by washing the column with wash buffer, and bound proteins were eluted in $0.5 \mathrm{ml}$ fractions with wash buffer containing $150 \mathrm{mM}$ galactose. The bound fraction was dialysed, lyophilized and analysed by SDS-PAGE and the protein components in the gel were visualized with either silver or Coomassie blue staining as described previously (Yang et al., 1997).

The functional role of the GBP was determined in adhesion assays as described above. Briefly, the GBP was pre-incubated with HBMECs for $30 \mathrm{~min}$, followed by the addition of $B$. mandrillaris.

To determine whether the GBP was expressed on cell-surface membranes, intact amoebae were incubated with a surface-impermeable reagent [0.5 mg EZ-Link Sulfo-NHS-Biotin (Pierce) in $1 \mathrm{ml}$ PBS for $30 \mathrm{~min}$ at room temperature] (Yang et al., 1997) that covalently attaches biotin to proteins on the outer surface of intact cells, followed by lysis of amoebae and extraction of the GBP as described above. The biotinylated proteins eluted from the column were electrophoresed and blotted onto nitrocellulose membranes. The membranes were blocked using blocking buffer $[25 \mathrm{mM}$ Tris/ $\mathrm{HCl}$ (pH 7.4), $150 \mathrm{mM} \mathrm{NaCl}, 0.1 \%$ Tween 20] containing $4 \%$ skimmed milk for $60 \mathrm{~min}$ at $22{ }^{\circ} \mathrm{C}$. Next, the biotinylated proteins were visualized using a Vectastain $\mathrm{ABC}-\mathrm{AmP}$ kit (Vector Laboratories) for $1 \mathrm{~h}$. Finally, blots were washed and developed using an enhanced chemiluminescence kit (Pharmacia Biosciences).

\section{RESULTS AND DISCUSSION}

\section{B. mandrillaris exhibits binding to HBMECs in a galactose-inhibitable manner}

To determine whether the carbohydrate moieties on the surface of the HBMECs are involved in B. mandrillaris interactions, adhesion assays were performed in the presence of the saccharides $\alpha$-mannose, xylose, glucose, fucose and $\beta$-galactose (final concentrations of 10,50 and $100 \mathrm{mM}$ ). The results revealed that B. mandrillaris exhibited greater than $90 \%$ binding to HBMECs and, of the various saccharides tested, only exogenous galactose inhibited binding of amoebae to HBMECs in a concentration-dependent manner (Fig. 1). Of interest, B. mandrillaris bound to microtitre wells coated with neoglycoprotein galactose-BSA but not to mannose-BSA-coated wells (data not shown). To confirm further that, at least primarily, $B$. mandrillaris recognized carbohydrate moieties on the 


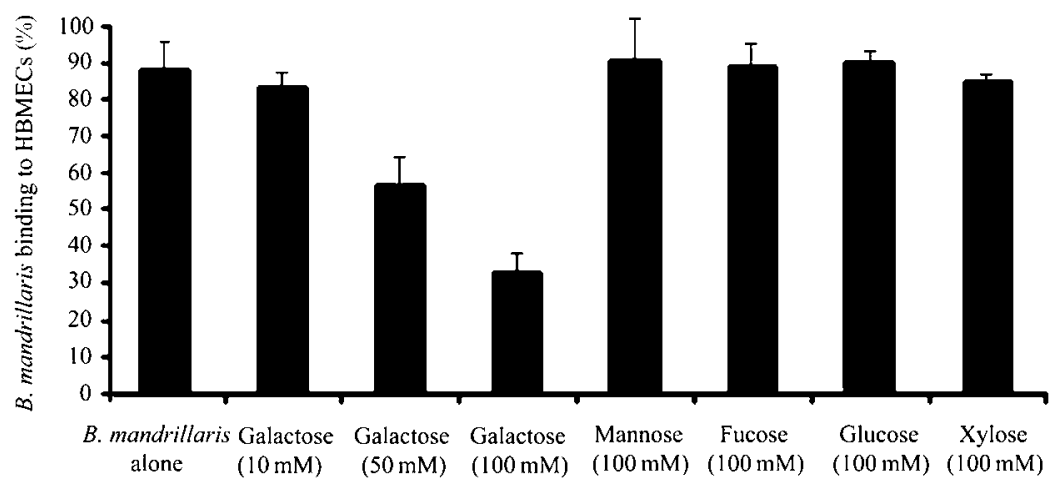

Fig. 1. B. mandrillaris exhibits binding to HBMECs in a galactose-inhibitable manner. To determine whether carbohydrate moieties on the surface of HBMECs are involved in $B$. mandrillaris interactions, adhesion assays were performed in the presence of various saccharides as described in Methods. Briefly, confluent monolayers of HBMECs were incubated with $B$. mandrillaris $\left(5 \times 10^{5}\right.$ amoebae per well). Plates were incubated at $37{ }^{\circ} \mathrm{C}$ in a $5 \% \mathrm{CO}_{2}$ incubator for $1 \mathrm{~h}$ and bound amoebae were enumerated. To test the effect of saccharides, assays were performed in the presence of various saccharides [ $\alpha-\mathrm{D}-$ mannopyranoside ( $\alpha$-mannose), xylose, glucose, fucose and $\beta$-galactose at final concentrations of 10, 50 and $100 \mathrm{mM}$ ]. Note that $B$. mandrillaris exhibited greater than $90 \%$ binding to HBMECs and, of the various saccharides, only exogenous galactose inhibited binding of amoebae to HBMECs in a concentration-dependent manner. The results represent the mean of three independent experiments performed in duplicate.

surface of the HBMECs, adhesion assays were performed using acetone-fixed amoebae or HBMECs. Acetone fixation denatures proteins but does not affect saccharides. As shown in Fig. 2, fixation of amoebae, but not of HBMECs, abolished B. mandrillaris adhesion to the host cells, suggesting that the molecular binding partners were protein(s) of the parasite and carbohydrate chains of plasma membrane glycoconjugates of the host cells.

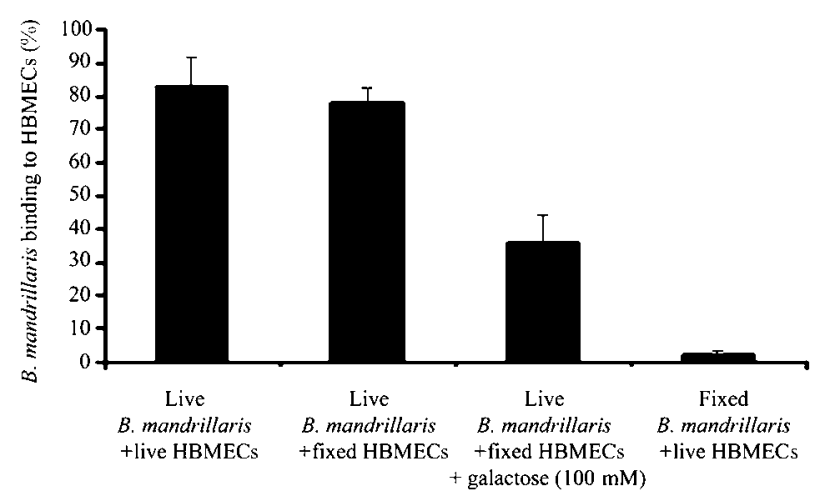

Fig. 2. To determine whether B. mandrillaris recognizes carbohydrate moieties on the surface of HBMECs, adhesion assays were performed using acetone-fixed amoebae or HBMECs as described in Methods. Briefly, confluent monolayers of HBMECs or amoebae were fixed with $100 \%$ ice-cold acetone and adhesion assays were performed as described in Fig. 1. Acetone fixation of amoebae, but not of HBMECs, abolished B. mandrillaris adhesion to the host cells, suggesting that the molecular binding partners are protein(s) of the parasite and carbohydrate chains of plasma membrane glycoconjugates of the host cells. The results represent the mean of three independent experiments performed in duplicate.

\section{Exogenous galactose has no effect on $B$. mandrillaris-mediated HBMEC cytotoxicity}

Previous studies have shown that Entamoeba histolytica adhesion to host cells can be blocked using exogenous galactose/ $\mathrm{N}$-acetylgalactosamine, which also inhibits parasite-mediated host-cell cytotoxicity (reviewed by Pillai \& Kain, 2000; Ravdin, 1989). These findings suggest that $E$. histolytica-mediated host-cell death is a contact-dependent event. As exogenous galactose inhibited B. mandrillaris adhesion to the host cells, we next determined the effects of galactose on B. mandrillaris-mediated HBMEC cytotoxicity. Our findings revealed that exogenous galactose had no significant effect on $B$. mandrillaris-mediated HBMEC cytotoxicity. In the absence of sugars, B. mandrillaris produced $73 \pm 7 \%$ HBMEC cytotoxicity, whilst the presence of exogenous sugars had no significant effect: amoebae produced $78.5 \pm 0.5 \%$ HBMEC cytotoxicity in the presence of $100 \mathrm{mM}$ galactose, $76 \pm 8.4 \%$ with $100 \mathrm{mM}$ mannose, $78.5 \pm 9.1 \%$ with $100 \mathrm{mM}$ fucose, $81.5 \pm 9.1 \%$ with $100 \mathrm{mM}$ glucose and $74.5 \pm 6.3 \%$ with $100 \mathrm{mM}$ xylose.

\section{B. mandrillaris expresses a GBP}

To determine whether B. mandrillaris expressed a GBP, cell lysates were analysed by chromatography on a galactoseSepharose column. Proteins that specifically bound to the affinity column were eluted with $150 \mathrm{mM}$ galactose and analysed by SDS-PAGE. We observed a major component in the bound fraction with an approximate molecular mass of $100 \mathrm{kDa}$ (Fig. 3a), suggesting that B. mandrillaris expresses a GBP (Fig. 3a). Next, to determine whether the GBP was membrane-associated, cell-surface proteins of intact $B$. mandrillaris were biotinylated, followed by 
isolation of the GBP on a galactose-Sepharose column and blotting using a Vectastain ABC-AmP kit as described in Methods. We observed a biotin-labelled component of the same molecular mass (approx. $100 \mathrm{kDa}$ ) as that found in the bound fraction of the unlabelled amoebae lysates (Fig. $3 \mathrm{~b})$. We also found another protein with an approximate molecular mass of $70 \mathrm{kDa}$. Overall, these findings suggested that the GBP is expressed on the cell-surface membranes of $B$. mandrillaris.

\section{The GBP inhibits $B$. mandrillaris binding to and cytotoxicity of HBMECs}

To determine the functional role of the GBP, adhesion assays were performed by pre-incubating HBMECs with the GBP. Our finding revealed that the GBP inhibited greater than $50 \%$ of $B$. mandrillaris adhesion to HBMECs (Fig. 4a) $(P<0.05$, using a paired $t$-test with one-tailed distribution), clearly indicating that the GBP expressed on the surface of parasites plays an important role in $B$.

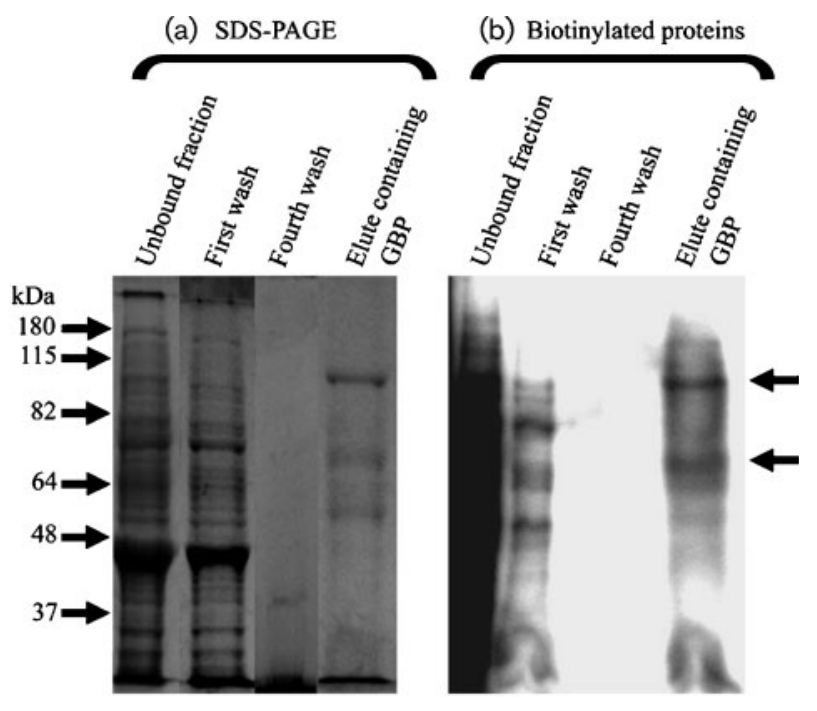

Fig. 3. B. mandrillaris expresses a GBP. (a) To determine whether B. mandrillaris expresses a GBP, cell lysates were analysed by chromatography on a galactose-Sepharose column followed by SDS-PAGE as described in Methods. Briefly, B. mandrillaris cell pellets $\left(10^{8}\right.$ trophozoites) were lysed and disrupted with a sonifier. The amoebae extracts were clarified by centrifugation at $500000 \mathrm{~g}$ for $1 \mathrm{~h}$ at $4{ }^{\circ} \mathrm{C}$ and analysed on a galactose-Sepharose column. Unbound proteins were removed and bound proteins were eluted with $150 \mathrm{mM}$ galactose. The bound fraction was dialysed, lyophilized and analysed by SDS-PAGE. A major component was detected in the bound fraction with an approximate molecular mass of $100 \mathrm{kDa}$. (b) To determine whether GBP is membraneassociated, cell-surface proteins of intact $B$. mandrillaris were biotinylated as described in Methods, followed by extraction of the GBP as above. A biotin-labelled component of the same molecular mass (approx. $100 \mathrm{kDa}$ ) as that found in the bound fraction of the unlabelled amoebae lysates was observed. The results are representative of three independent experiments. mandrillaris adhesion to HBMECs. In contrast, the unbound fraction had no significant effect on binding of amoebae to HBMECs (Fig. 4a).

Next, to determine the effects of the GBP on $B$. mandrillaris-mediated HBMEC cytotoxicity, cytotoxicity assays were performed in the presence of the GBP. As shown in Fig. 4(b), the results revealed that the GBP inhibited B. mandrillaris-mediated HBMEC cytotoxicity $(P$ $<0.05$ using a paired $t$-test with one-tailed distribution). Overall, these results suggested that the GBP may play important roles in B. mandrillaris interactions with HBMECs.

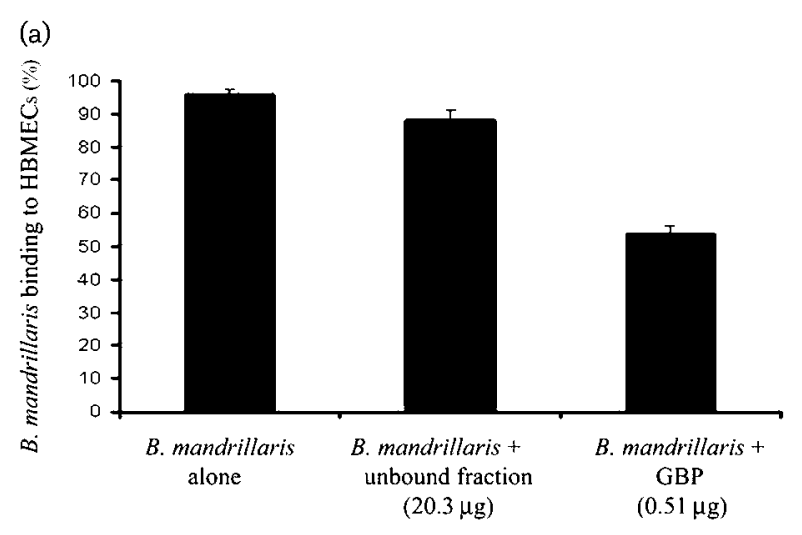

(b)

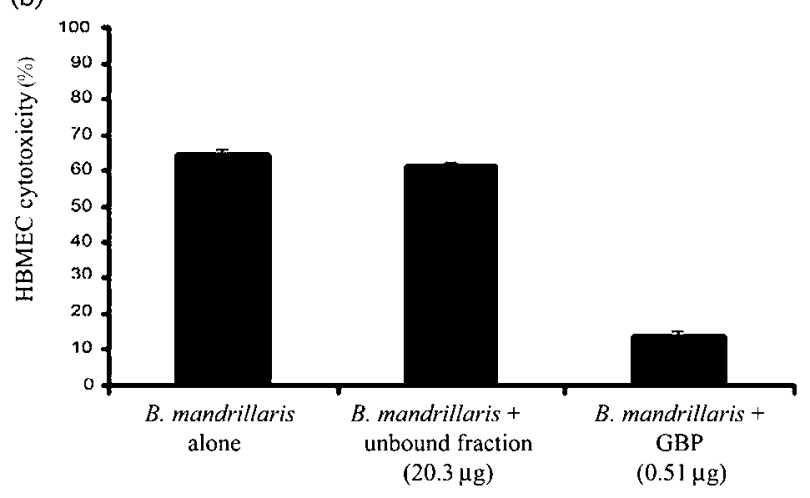

Fig. 4. GBP inhibits $B$. mandrillaris binding to and cytotoxicity towards HBMECs. (a) To determine the functional role of the GBP, adhesion assays were performed by pre-incubating HBMECs with the GBP as described in Methods. The GBP inhibited more than $50 \%$ of $B$. mandrillaris adhesion to HBMECs $(P<0.05$, using a paired $t$-test with one-tailed distribution). In contrast, the unbound fraction had no significant effect on binding of amoebae to HBMECs. (b) To determine the effects of the GBP on $B$. mandrillaris-mediated HBMEC death, cytotoxicity assays were performed in the presence of the GBP as described in Methods. Briefly, HBMECs were incubated with the GBP for $30 \mathrm{~min}$ followed by inoculation of $B$. mandrillaris. Plates were then incubated for up to $24 \mathrm{~h}$. At the end of the incubation period, supernatants of co-cultures of $B$. mandrillaris and HBMECs were collected and the percentage of $\mathrm{LDH}$ release was determined. The results showed that the GBP inhibited $B$. mandrillaris-mediated HBMEC cytotoxicity. 
One of the key steps in the pathogenesis of BAE is $B$. mandrillaris invasion of the central nervous system, which probably occurs at sites of the blood-brain barrier. Using HBMECs, which constitute the blood-brain barrier, we have recently shown that $B$. mandrillaris produces HBMEC cytotoxicity, which may lead to blood-brain barrier perturbations (Matin et al., 2006, 2007). However, the underlying molecular mechanisms associated with amoebae traversal of the blood-brain barrier leading to pathological features remain unclear. Although the successful traversal of $B$. mandrillaris across the blood-brain barrier may require multiple events, we hypothesize that adhesion is a primary step in amoebae transmigration of the HBMECs. In this study, we demonstrated that $B$. mandrillaris binds to HBMECs in a galactose-inhibitable manner and identified a GBP expressed on the surface of $B$. mandrillaris. The inhibition of $B$. mandrillaris adhesion to HBMECs was specific to galactose, as other saccharides had no effect on amoebae binding. To confirm further that the galactose-containing glycoproteins on HBMECs serve as attachment sites for B. mandrillaris, adhesion assays were performed by fixing either amoebae or HBMECs. We observed that acetone fixation of amoebae abolished $B$. mandrillaris binding to HBMECs, whilst amoebae bound to acetone-fixed HBMECs in a galactose-inhibitable manner, suggesting that the parasite binds to galactose residues on HBMECs. The presence of a GBP in $B$. mandrillaris has recently been suggested by Rocha-Azevedo et al. (2007), who demonstrated that B. mandrillaris binds to laminin and that these interactions can be inhibited using exogenous galactose. Our results support these findings and have identified the expression of a GBP on the surface membranes of $B$. mandrillaris. However, given the complexity of host-parasite interactions, it is tempting to speculate that the aforementioned interactions only provide initial attachment, which is likely to be followed by closer associations with a more intimate contact of B. mandrillaris to HBMECs involving the GBP as well as other adhesin(s). Such binding is probably necessary to withstand blood flow, as well as for subsequent crossing of the blood-brain barrier. Further studies are needed to validate the concept of other determinants, in addition to GBP, and their roles in B. mandrillaris-HBMEC interactions.

Using chromatography of extracts of B. mandrillaris on a galactose-Sepharose column, we isolated a GBP with an approximate molecular mass of $100 \mathrm{kDa}$. Previous studies have identified a galactose $/ \mathrm{N}$-acetylgalactosamine lectin in Entamoeba. The isolation and characterization of this amoebic lectin revealed that it was a $260 \mathrm{kDa}$ heterodimer, composed of a $170 \mathrm{kDa}$ heavy subunit and a $31 / 35 \mathrm{kDa}$ light subunit that is anchored by glycosylphosphatidylinositol to the surface membrane bilayer (reviewed by Pillai \& Kain, 2000; Ravdin, 1989). Interestingly, Ravdin \& Guerrant (1981) showed that the E. histolytica lectin is important in adherence and contact-dependent cytolysis of human cells. This was shown using exogenous galactose/ $\mathrm{N}$-acetylgalactosamine, which blocked $E$. histolytica binding to and killing of target cells, suggesting that E. histolytica-mediated human cell cytotoxicity is a contact-dependent process. In contrast, our findings revealed that, although exogenous galactose blocked $B$. mandrillaris binding to HBMECs, it had no effect on $B$. mandrillaris-mediated HBMEC death. We are cautious in interpreting these findings as our cytotoxicity assays were performed by incubating B. mandrillaris with HBMECs over longer periods of time, i.e. $24 \mathrm{~h}$. Of interest, the GBP inhibited B. mandrillaris-mediated HBMEC cytotoxicity, suggesting the involvement of diverse mechanisms. Overall, these findings suggest that $B$. mandrillarismediated HBMEC cytotoxicity may involve both contact-dependent and -independent mechanisms. Future studies are in progress to address these issues.

In conclusion, we have shown for the first time that $B$. mandrillaris primarily binds to galactose residues of the plasma membrane glycoconjugates of the host cells and identified a GBP expressed on the cell surface of these parasites. Further studies will characterize the GBP and determine its potential role in $B$. mandrillaris pathogenesis, which may identify novel targets for the rational development of therapeutic interventions and/or design of preventative strategies. This is not a novel concept. For example, Acanthamoeba, a close relative of Balamuthia, is a causative agent of blinding keratitis. Recent studies have shown that Acanthamoeba binds to corneal epithelial cells using its mannose-binding protein (Garate et al., 2004; Morton et al., 1991; Yang et al., 1997). Interestingly, oral immunization with recombinant mannose-binding protein protected against Acanthamoeba keratitis in vivo. Similar strategies may be developed against $\mathrm{BAE}$ and the identification of the GBP should lay a foundation for future studies.

\section{ACKNOWLEDGEMENTS}

This work was supported by grants from Birkbeck Life Sciences Faculty and The Royal Society.

\section{REFERENCES}

Alsam, S., Kim, K. S., Stins, M., Rivas, A. O., Sissons, J. \& Khan, N. A. (2003). Acanthamoeba interactions with human brain microvascular endothelial cells. Microb Pathog 35, 235-241.

Booton, G. C., Schuster, F. L., Carmichael, J. R., Fuerst, P. A. \& Byers, T. J. (2003a). Balamuthia mandrillaris: identification of clinical and environmental isolates using genus-specific PCR. J Eukaryot Microbiol 50, 508-509.

Booton, G. C., Carmichael, J. R., Visvesvara, G. S., Byers, T. J. \& Fuerst, P. A. (2003b). Identification of Balamuthia mandrillaris by PCR assay using the mitochondrial $16 \mathrm{~S}$ rRNA gene as a target. J Clin Microbiol 41, 453-455.

Garate, M., Cao, Z., Bateman, E. \& Panjwani, N. (2004). Cloning and characterization of a novel mannose-binding protein of Acanthamoeba. J Biol Chem 279, 29849-29856. 
Garate, M., Marchant, J., Cubillos, I., Cao, Z., Khan, N. A. \& Panjwani, N. (2006). In vitro pathogenicity of Acanthamoeba is associated with the expression of the mannose-binding protein. Invest Ophthalmol Vis Sci 47, 1056-1062.

Hostetter, M. K. (1994). Adhesins and ligands involved in the interaction of Candida spp. with epithelial and endothelial surfaces. Clin Microbiol Rev 7, 29-42.

Huang, Z. H., Ferrante, A. \& Carter, R. F. (1999). Serum antibodies to Balamuthia mandrillaris, a free-living amoeba recently demonstrated to cause granulomatous amoebic encephalitis. J Infect Dis 179, 1305-1308.

Jayasekera, S., Sissons, J., Tucker, J., Rogers, C., Nolder, D., Warhurst, D., Alsam, S., White, J. M. L., Higgins, E. M. \& Khan, N. A. (2004). Post mortem culture of Balamuthia mandrillaris from the brain and cerebrospinal fluid of a case of granulomatous amoebic meningoencephalitis, using human brain microvascular endothelial cells. J Med Microbiol 53, 1007-1012.

Karlsson, K. A. (1989). Animal glycosphingolipids as membrane attachment sites for bacteria. Annu Rev Biochem 58, 309-350.

Kiderlen, A. F., Tata, P. S., Ozel, M., Laube, U., Radam, E. \& Schafer, H. (2006). Cytopathogenicity of Balamuthia mandrillaris, an opportunistic causative agent of granulomatous amebic encephalitis. J Eukaryot Microbiol 53, 456-463.

Martinez, A. J., Schuster, F. L. \& Visvesvara, G. S. (2001). Balamuthia mandrillaris: its pathogenic potential. J Eukaryot Microbiol (Suppl.), 6S-9S.

Matin, A., Stins, M., Kim, K. S. \& Khan, N. A. (2006). Balamuthia mandrillaris exhibit metalloprotease activities. FEMS Immunol Med Microbiol 47, 83-91.

Matin, A., Jeong, S. R., Stins, M. \& Khan, N. A. (2007). Effects of human serum on Balamuthia mandrillaris interactions with the human brain microvascular endothelial cells. J Med Microbiol 56, 30-35.

Morton, L. D., McLaughlin, G. L. \& Whiteley, H. E. (1991). Effect of temperature, amebic strain and carbohydrates on Acanthamoeba adherence to corneal epithelium in vitro. Infect Immun 59, 3819-3822.

Pillai, D. R. \& Kain, K. C. (2000). Recent developments in amoebiasis: the Gal/GalNAc lectins of Entamoeba histolytica and Entamoeba dispar. Microbes Infect 2, 1775-1783.

Qvarnstrom, Y., Visvesvara, G. S., Sriram, R. \& da Silva, A. J. (2006). Multiplex real-time PCR assay for simultaneous detection of Acanthamoeba spp., Balamuthia mandrillaris, and Naegleria fowleri. J Clin Microbiol 44, 3589-3595.
Ravdin, J. I. (1989). Entamoeba histolytica: from adherence to enteropathy. J Infect Dis 159, 420-429.

Ravdin, J. I. \& Guerrant, R. L. (1981). Role of adherence in cytopathogenic mechanisms of Entamoeba histolytica. Study with mammalian tissue culture cells and human erythrocytes. J Clin Invest 68, 1305-1313.

Rocha-Azevedo, B., Jamerson, M., Cabral, G. A., Silva-Filho, F. C. \& Marciano-Cabral, F. (2007). The interaction between the amoeba Balamuthia mandrillaris and extracellular matrix glycoproteins in vitro. Parasitology 134, 51-58.

Schuster, F. L. \& Visvesvara, G. S. (2004). Free-living amoebae as opportunistic and non-opportunistic pathogens of humans and animals. Int J Parasitol 34, 1001-1027.

Sissons, J., Kim, K. S., Stins, M., Jayasekera, S., Alsam, S. \& Khan, N. A. (2005). Acanthamoeba castellanii induces host cell death via a phosphatidylinositol 3-kinase-dependent mechanism. Infect Immun 73, 2704-2708.

Sissons, J., Alsam, S., Stins, M., Rivas, A. O., Morales, J. L., Faull, J. \& Khan, N. A. (2006). Use of in vitro assays to determine effects of human serum on biological characteristics of Acanthamoeba castellanii. J Clin Microbiol 44, 2595-2600.

Stins, M. F., Gilles, F. \& Kim, K. S. (1997). Selective expression of adhesion molecules on human brain microvascular endothelial cells. $J$ Neuroimmunol 76, 81-90.

Tavares, M., Correia da Costa, J. M., Carpenter, S. S., Santos, L. A., Afonso, C., Aguiar, A., Pereira, J., Cardoso, A. I., Schuster, F. L. \& other authors (2006). Diagnosis of first case of Balamuthia amoebic encephalitis in Portugal by immunofluorescence and PCR. J Clin Microbiol 44, 2660-2663.

Visvesvara, G. S., Martinez, A. J., Schuster, F. L., Leitch, G. J., Wallace, S. V., Sawyer, T. K. \& Anderson, M. (1990). Leptomyxid ameba, a new agent of amebic meningoencephalitis in humans and animals. J Clin Microbiol 28, 2750-2756.

Visvesvara, G. S., Schuster, F. L. \& Martinez, A. J. (1993). Balamuthia mandrillaris, N. G., N. Sp., agent of amebic meningoencephalitis in humans and other animals. J Eukaryot Microbiol 40, 504-514.

Yagi, S., Booton, G. C., Visvesvara, G. S. \& Schuster, F. L. (2005). Detection of Balamuthia mitochondrial 16S rRNA gene DNA in clinical specimens by PCR. J Clin Microbiol 43, 3192-3197.

Yang, Z., Cao, Z. \& Panjwani, N. (1997). Pathogenesis of Acanthamoeba keratitis: carbohydrate-mediated host-parasite interactions. Infect Immun 65, 439-445. 